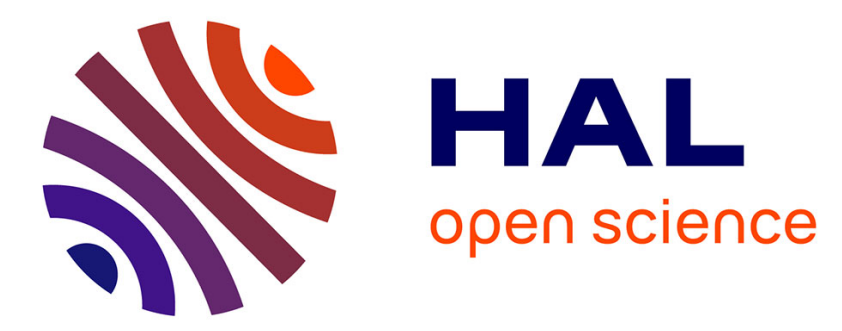

\title{
Analysis of individual terrestrial gamma-ray flashes with lightning leader models and Fermi Gamma-ray Burst Monitor data
}

B. G. Mailyan, Wei Xu, Sébastien Celestin, M.S. Briggs, J. R. Dwyer, E. S.

Cramer, O. J. Roberts, M. Stanbro

\section{To cite this version:}

B. G. Mailyan, Wei Xu, Sébastien Celestin, M.S. Briggs, J. R. Dwyer, et al.. Analysis of individual terrestrial gamma-ray flashes with lightning leader models and Fermi Gamma-ray Burst Monitor data. Journal of Geophysical Research Space Physics, 2019, 124 (8), pp.7170-7183. 10.1029/2019JA026912 . insu-02263534

\section{HAL Id: insu-02263534 \\ https://hal-insu.archives-ouvertes.fr/insu-02263534}

Submitted on 6 Sep 2019

HAL is a multi-disciplinary open access archive for the deposit and dissemination of scientific research documents, whether they are published or not. The documents may come from teaching and research institutions in France or abroad, or from public or private research centers.
L'archive ouverte pluridisciplinaire HAL, est destinée au dépôt et à la diffusion de documents scientifiques de niveau recherche, publiés ou non, émanant des établissements d'enseignement et de recherche français ou étrangers, des laboratoires publics ou privés. 


\section{JGR Space Physics}

\section{RESEARCH ARTICLE \\ 10.1029/2019JA026912 \\ Key Points: \\ Analysis of Individual Terrestrial Gamma-Ray Flashes With Lightning Leader Models and Fermi Gamma-Ray Burst Monitor Data}

- Fermi GBM individual TGF spectra are analyzed using lightning leader models of various leader potentials, beaming tilts, and source altitudes

- Lightning leader models with potentials of $200 \mathrm{MV}$ and tilted beams often best fit the data

- A few, exceptionally soft TGFs can be well explained using 60-MV lightning leaders with tilted beams

Correspondence to: B. G. Mailyan and W. Xu, bagrat.mailyan@uah.edu; Wei-Xu@colorado.edu

Citation:

Mailyan, B. G., Xu, W., Celestin, S., Briggs, M. S., Dwyer, J. R.

Cramer, E. S., et al. (2019). Analysis of individual terrestrial gamma-ray flashes with lightning leader models and Fermi Gamma-ray Burst Monitor data. Journal of Geophysical Research: Space Physics, 124. https://doi.org/10. 1029/2019JA026912

Received 1 MAY 2019

Accepted 16 JUL 2019

Accepted article online 1 AUG 2019

(C)2019. American Geophysical Union. All Rights Reserved.

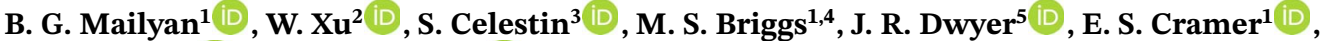 \\ O. J. Roberts ${ }^{6}$ iD, and M. Stanbro4 ${ }^{4}$ \\ ${ }^{1}$ Center for Space Plasma and Aeronomic Research, The University of Alabama in Huntsville, Huntsville, AL, USA, \\ ${ }^{2}$ Department of Aerospace Engineering Sciences, University of Colorado Boulder, Boulder, CO, USA, ${ }^{3}$ LPC2E, \\ University of Orleans, CNRS, Orleans, France, ${ }^{4}$ Department of Space Science, The University of Alabama in Huntsville, \\ Huntsville, AL, USA, ${ }^{5}$ Department of Physics and Space Science Center (EOS), University of New Hampshire, Durham, \\ NH, USA, ${ }^{6}$ Science and Technology Institute, Universities Space Research Association, Huntsville, AL, USA
}

Abstract The Gamma-ray Burst Monitor (GBM) onboard the Fermi spacecraft has observed many tens of sufficiently bright events, which are suitable for individual analysis. In our previous study, we fit individual, bright terrestrial gamma-ray flashes (TGFs) with Relativistic Runaway Electron Avalanche (RREA) models for the first time. For relativistic-feedback-based models, the TGF-producing electrons, which are seeded internally by a positive feedback effect, are usually accelerated in a large-scale field with fully developed RREAs. Alternatively, lightning leader models may apply to either a large-scale thunderstorm fields with fully developed RREAs or to inhomogeneous fields in front of lightning leaders where RREAs only develop partially. The predictions of the latter, inhomogeneous models for the TGF-beaming geometry show some differences from estimations of the relativistic feedback models in homogeneous fields. In this work, we analyze a large sample of 66 bright Fermi GBM TGFs in the framework of lightning leader models, making comparisons with previous results from the homogeneous-field RREA models. In most cases, the spectral analysis does not strongly favor one mechanism over the other, with 59\% of the TGF events being best fit with the fully developed RREA mechanism, which corresponds to high-potential leader models. The majority of the GBM-measured TGFs can be best fit if the source altitude is below $15 \mathrm{~km}$ and $70 \%$ of events best fit by leader models cannot be satisfactorily modeled unless a tilted photon beam is used. For several spectrally soft TGFs, the tilted beam low-potential leader model can best fit the data.

\section{Introduction}

Terrestrial gamma-ray flashes (TGFs) are submillisecond bursts of photons observed by various space missions at altitudes of 500-600 km (Briggs et al., 2010; Fishman et al., 1994; Marisaldi et al., 2010; Smith et al., 2005). TGF photon energy spectra can reach tens of megaelectron volts. Despite the high rates of incident photons occurring over a submillisecond timescale, each individual TGF has a relatively small number of counts. This makes the analysis of individual TGFs challenging as typically the statistics are not good enough to constrain the TGF production models based on individual events. That is why traditionally cumulative spectra of TGFs are studied by adding counts from different events to have sufficient data to constrain the models (Dwyer \& Smith, 2005; Tavani et al., 2011).

Extensive analysis of cumulative TGF energy spectra focused on quantifying the typical characteristics of TGF sources (including the spectral hardness and production altitude), have been previously studied. However, the variation of individual TGF events remains insufficiently investigated, which represents a major goal of this study. Gjesteland et al. (2015) analyzed three bright TGFs observed by the Reuven Ramaty High Energy Solar Spectroscopic Imager above the Mediterranean Sea region. For their brightest event, it was not possible to get constraints on the source altitude of the TGF within 6- to 14-km region using a $\chi^{2}$ method. More recently, Mailyan et al. (2016) have done a spectral analysis of more than 40 events observed by the Gamma-ray Burst Monitor (GBM) on-board the Fermi spacecraft (Meegan et al., 2009). The likelihood analysis showed that the individual spectra are diverse and that different TGF events are best fit by various 
altitude and photon beam geometry models. Analysis of cumulative TGF spectra is potentially problematic, as the cumulative spectrum will merge spectra of different shapes from TGFs with different characteristics.

Currently, two classes of models are proposed to explain the observations of TGFs. In one of the models, today known as relativistic feedback model, electrons accelerate in large-scale electric fields within thunderclouds followed by bremsstrahlung emission of photons (Dwyer, 2003). The other model considers the thermal runaway electrons produced at the tips of lightning leaders and accelerated in either the leader-produced field or the large-scale thunderstorm field (Celestin \& Pasko, 2011; Dwyer, 2008; Moss et al., 2006). Both models make use of relativistic runaway electron avalanches (RREAs) to involve electron multiplication.

While analyzing the TGF data, care should be taken to account for spectral distortions caused by the pulse pileup, as those instrumental effects play an important role for TGFs with high photon rates. Not accounting for these effects may lead to erroneous interpretation of the data (see discussion in Mailyan et al., 2016, and references therein).

Mailyan et al. (2016) found that several events were best fit by wide geometry beams, while some other events were best fit by narrow beams. The best fit with narrow beams, where wider beams are excluded, would seem to reject lightning leader scenario. This is because the source photon beam produced by lightning leaders is naturally broad (Xu et al., 2012) since the angular distribution of source photons is determined by the configuration of electric field produced by lightning leaders in the tip region. However, lightning leader models were not actually used in our previous work, and that conclusion would be premature. In this work we expand the set of models used in Mailyan et al. (2016) to also include lightning leader models in inhomogeneous fields. Tilted beams were also included (only for the lightning leader models) as recent radio measurements have revealed that the beam of source bremsstrahlung photons is likely tilted (Lyu et al., 2016). There were some indications of TGF producing beams being tilted from satellite TGF measurements as well (Fitzpatrick et al., 2014; Mailyan et al., 2016). Variable photon rates are also considered at the spacecraft altitude to account for the pulse pileup effects. Primarily focusing on the lightning leader model of the TGF production, we study the beaming geometry issues discussed in Mailyan et al. (2016) and spectra variability effects discussed in Celestin et al. (2015) using the Fermi GBM TGF data.

\section{Methodology}

\subsection{Data Sources}

The GBM instrument is made up of 12 thallium-doped sodium iodide ( $\mathrm{NaI}(\mathrm{Tl})$ ) and two bismuth germanate (BGO) detectors (Meegan et al., 2009). In this paper, we analyze the TGF spectra using the data from the BGO detectors, which have a broad spectral response $(0.2-40 \mathrm{MeV})$ and large effective area $\left(\sim 160 \mathrm{~cm}^{2}\right.$; Tierney et al., 2013). The BGOs are positioned on opposite sides of the spacecraft. The photon counts are assigned to spectral channels according to 128 pseudo-logarithmically spaced energy channels, and time-tagged with relative timing resolution of $2 \mu \mathrm{s}$. The relative timing resolution of $2 \mu \mathrm{s}$ is critical for studying submillisecond bursts like TGFs. The nominal dead time is $2.6 \mu \mathrm{s}$. However, if a count is registered in the overflow channel (>40 MeV for the BGO detectors), the dead time is $10.4 \mu \mathrm{s}$. We focus on bright TGFs which have very low frequency (VLF) radio associations, as described in Mailyan et al. (2016) and Roberts et al. (2018). TGFs without a VLF association may occur anywhere within about $800 \mathrm{~km}$ of the subspacecraft position. For TGFs with a VLF association, the VLF geolocation provides a position accuracy of $\sim 10 \mathrm{~km}$, which makes it possible to precisely calculate the detector response and is mandatory in interpreting the gamma-ray spectra.

The TGF detection rate significantly increased after new data collection modes and analysis methods were introduced in 2012. These include an updated onboard triggering algorithm and off-line search algorithms of the high time resolution data (Briggs et al., 2013; Roberts et al., 2018). At the same time, the efficiency of the radio associations was significantly increased due to the upgrades of the World Wide Lightning Location Network (Hutchins et al., 2012). Having these improvements, we are able to study an unprecedentedly large set of 66 bright TGFs having more than 20 counts in either of BGO detectors. By TGF brightness, we mean the number of photon counts observed by the GBM and not the intrinsic brightness at the source. Also, it is worth stating that especially short-duration intrinsically bright TGFs may have a small number of observed photon counts due to instrumental effects. For the sake of comparison, all the TGF events analyzed in Mailyan et al. (2016) have been reinvestigated in the context of the lightning leader models in the present work. 


\subsection{Lightning Leader Models}

In this paper, the analysis of individual TGF events measured by Fermi GBM is primarily based on a suite of Monte Carlo models describing the TGF production by lightning leaders, for both electrons (Celestin \& Pasko, 2011) and photons (Xu et al., 2012). The modeling procedure is similar to that used in, for example, Celestin et al. (2012) and Xu et al. (2015), specifically in three steps. First, we calculate the electric field produced near the tip region of the negative end of lightning leaders using the method of moments. Second, using the Monte Carlo model for electrons, we simulate the acceleration of thermal runaway electrons in this leader field and the production of bremsstrahlung photons. We emphasize that the maximum electric field of $50 \mathrm{kV} / \mathrm{cm}$ (at ground level) is used in order to avoid the acceleration of runaway electrons in the extremely strong electric field obtained by the static vacuum solution (method of moments) at locations close to the leader tip (see discussion in Celestin \& Pasko, 2011; Celestin et al., 2015). This assumption underestimates the true potential drop (Skeltved et al., 2017). In this study, the parameter that impacts the energy of electrons the most is the potential drop in the leader tip region that is available for the acceleration of runaway electrons. Mallios et al. (2013) have shown that downward positive leaders propagating before the start of the propagation of negative leaders in a bidirectional intracloud discharge could lead to potential drops as high as 300 MV. Skeltved et al. (2017; Figure 5) have also found that potential drops similar to those used in the present study can be obtained by taking the horizontal development of positive and negative leaders into account. Simulating the full dynamics of the electric field and potential drop in the leader steamer zone system during the negative corona flash would improve the description of the physical relation between the leader and the electron acceleration processes at the cost of increasing complexity. The leader potential drops used in the present and previous studies should be seen as an underestimation of true potential drops in the considered leaders geometries. Finally, the energy and position of those bremsstrahlung photons obtained in the second step are directly input into the Monte Carlo model for photons, collecting photons that escape the Earth's atmosphere at $565 \mathrm{~km}$ altitude, that is, the mean altitude of Fermi's orbit. In the following, we introduce the initial parameters and numerical models used in the present study.

The potential difference between the tip of the lightning leader and the ambient potential (named "potential drop" hereafter for simplicity) is a critical parameter in this work because it directly controls the avalanche processes of thermal runaway electrons (see discussion in Celestin et al., 2015). In this study, we mainly focus on lightning leaders with three potential drops: 60, 100, and $200 \mathrm{MV}$. These values are chosen because the bremsstrahlung gamma rays originating from these leaders have similar total energies as typical satellite measurements (e.g., Celestin et al., 2015; Xu et al., 2012). This range of leader-accelerated potential drops is plausible (Mallios et al., 2013). Higher potential drops would lead to photon spectra close to the classical RREA spectrum obtained in a homogeneous field configuration (Celestin et al., 2015), which was already studied in Mailyan et al. (2016). This means that the spectral analysis solely is insufficient to distinguish the RREA mechanism from very high potential (>200 MV) lightning leader models. For each potential drop, we calculate the electric field near the leader tip region by assuming that the lightning leader is a perfectly conducting cylinder and is aligned with the large-scale thunderstorm electric field. With this assumption, we then use the method of moments (Balanis, 1989, p. 670) to convert the electric potential integral equation into a set of matrix equations and thereby solve the electric charge distribution induced on the lightning channel. The electric field produced in the vicinity of lightning leader tip is further reconstructed using the charge distribution obtained. It should be noted that the conductivity of the streamer zone is not taken into account and hence the dynamics of the electric field is not modeled. For this reason, the time dynamics of the TGF is not obtained with this model. That is justified by the fact that negative corona flashes initiate prior to the formation of a large streamer zone.

The potential drop in the leader tip region $U_{1}$ can be approximated by $U_{1}=E_{0} l / 2$ (Bazelyan \& Raizer, 2000, p. 54), where $E_{0}$ is the ambient large-scale thunderstorm electric field and $l$ is the length of the unbranched leader channel. For the three potential drops, we choose the following typical values for $E_{0}$ and $l: U_{1}=60 \mathrm{MV}$, $E_{0}=2 \mathrm{kV} / \mathrm{cm}, l=0.6 \mathrm{~km} ; U_{1}=100 \mathrm{MV}, E_{0}=0.5 \mathrm{kV} / \mathrm{cm}, l=4 \mathrm{~km} ; U_{1}=200 \mathrm{MV}, E_{0}=1 \mathrm{kV} / \mathrm{cm}, l=4 \mathrm{~km}$. In all cases, the radius of the leader channel is assumed to be $1 \mathrm{~cm}$ (Rakov \& Uman, 2003, Section 4.4.6, p. 134). Of note, the specific choices of $E_{0}$ and $l$ are not critical as long as the resultant potential drop is the same.

We use the Monte Carlo model developed by Celestin and Pasko (2011) in order to simulate the propagation and collisions of electrons in air. This model explicitly solves for the equation of electron motion in three-dimensional (3-D) velocity space and 3-D configuration space; it is relativistic and simulates electrons in the energy regime from subelectron volt to gigaelectron volt; the ionization collision, which is of crucial 


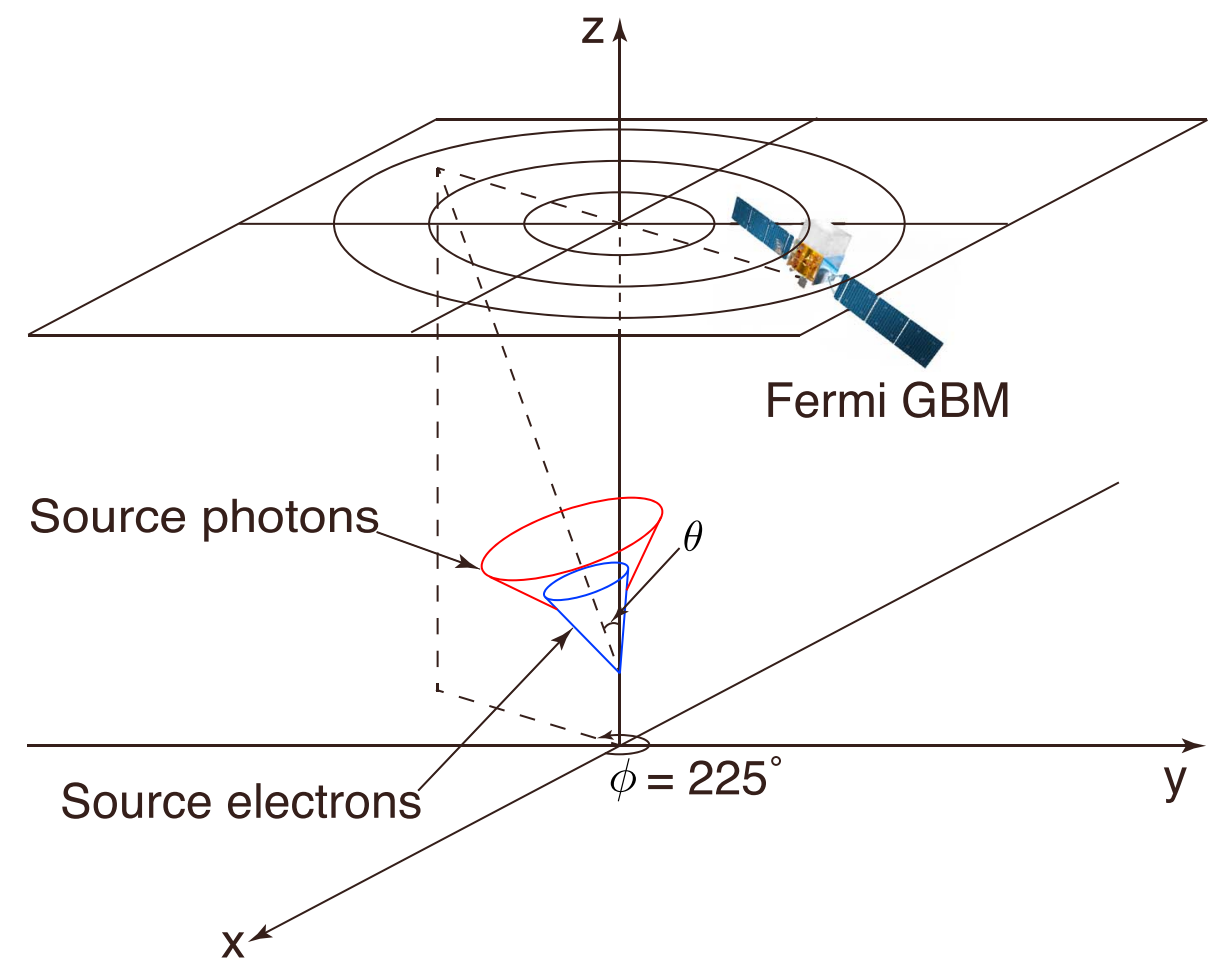

Figure 1. Illustration of the geometry used for simulating tilted beams of source bremsstrahlung photons. Tilted beam opposite to the spacecraft is illustrated. GBM = Gamma-ray Burst Monitor.

importance for modeling runaway processes, is simulated using the singly differential cross sections calculated via the relativistic binary-encounter-Bethe model (e.g., Celestin \& Pasko, 2010). In theory, thermal runaway electrons resulting from streamer discharges may have an energy close to $\sim 65 \mathrm{keV}$ (Celestin \& Pasko, 2011). Thus, this energy is assigned to the initial group of thermal runaway electrons used in Monte Carlo simulations. These electrons are initially placed at a location where the corresponding electric field is $50 \mathrm{kV} / \mathrm{cm}$, as reasonable for the field magnitude at the leader tip (Bazelyan \& Raizer, 2000, pp. 67-68).

The Monte Carlo model employed to simulate photon transport in the Earth's atmosphere is similar to that described in Østgaard et al. (2008). This model takes into account three main collision types for photons with energies between $10 \mathrm{keV}$ and $100 \mathrm{MeV}$ : photoelectric absorption, Compton scattering, and electron-positron pair production. We emphasize that this set of Monte Carlo models has been utilized to study RREA properties (e.g., Celestin et al., 2012), the acceleration of electrons in inhomogeneous fields produced by lightning leaders (e.g., Celestin et al., 2012; Xu et al., 2015), and optical emissions produced by TGFs (Xu et al., 2017); modeling results show good agreements with previously published results (also, see the review and discussion in Dwyer et al., 2012).

Lyu et al. (2016) have recently pointed out that the majority of the initial leader steps, as well as their associated electric fields and bremsstrahlung production processes, are tilted away from the vertical direction and this tilted leader stepping could affect TGF observation from space. Motivated by this finding, in the present study, we simulate TGF production by both vertical and tilted lightning leaders. Because the energy distribution of thermal runaway electrons is predominantly controlled by the potential drop of lightning leaders, the simulation results of electron acceleration (the first two steps as described above) are the same in these two scenarios. As for the third step of photon simulation, to mimic the tilted leader channel, the ensemble of bremsstrahlung photons obtained from electron simulations is rotated from the vertical direction using two angles: $\theta$ and $\phi$, similar to those defined in the spherical coordinate system. Figure 1 shows the geometry used in the simulation of tilted lightning leaders. $x y z$ directions in the simulation are arbitrary. Specifically, $\theta$ is the angle between the axis of the photon beam and $+z$ direction, and $\phi$ is the angle between $+x$ direction and the orthogonal projection of beam axis on the $x y$ plane. Lyu et al. (2016) have revealed that initial stepping leaders typically have a tilting angle between $6^{\circ}$ and $38^{\circ}$. The angle $\theta$ is chosen to be either 


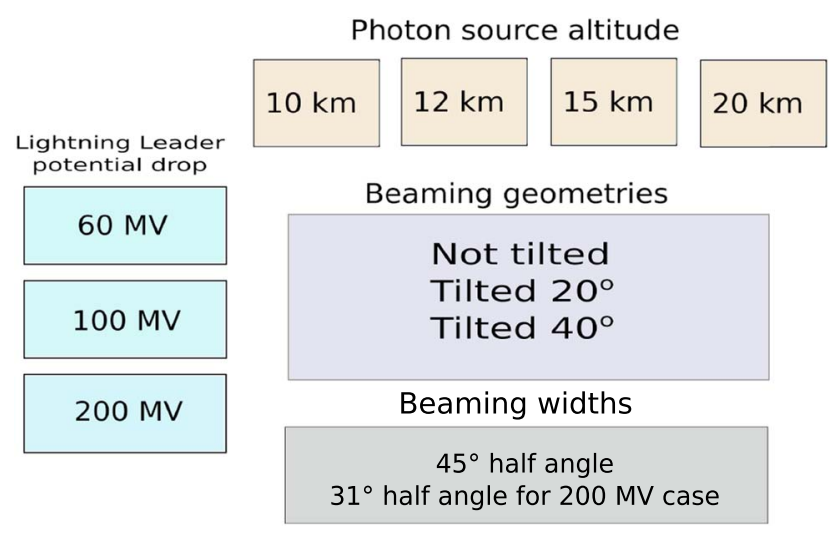

Figure 2. Diagram of the lightning leader models used in this work. Various leader potentials, source altitudes, beaming tilts, and widths are considered. $20^{\circ}$ or $40^{\circ}$ in the present work. The angle $\phi$ is assumed to be $225^{\circ}$ for both $\theta$ values and, for both vertical and tilted leaders, the half angle of the photon beam is assumed to be $45^{\circ}$ following previous studies (e.g., Carlson et al., 2007; Dwyer et al., 2012). The tilted geometry could help explain Fermi measurements at relatively close offset distances, but with unexpected low fluence and soft spectrum. A value of $225^{\circ}$ is used for the simplicity of photon collection. Source photons are assumed to be produced isotropically within a cone with a half angle of $45^{\circ}$. For 200-MV tilted leader case, we considered also a narrower beam, which assumes "natural" beam width having a half angle of $31^{\circ}$ to produce the hardest possible (for this leader potential value) spectra at small offset distances. This half angle is specifically derived from Monte Carlo simulation results. The variety of lightning leader models considered in this work is displayed in Figure 2. Likelihood analysis method is used to fit the TGF data using these models as described in Mailyan et al. (2016).

\subsection{The Time-Resolved Spectral Fitting}

The electron acceleration process in atmospheric electric fields is not an instantaneous process, and, as a result, TGFs at the source altitude are not produced instantaneously, but have a time distribution. Fitzpatrick et al. (2014) had shown that GBM observational data matches well with the models if one uses a source distribution of gamma-rays distributed by a Gaussian function with a sigma of $50 \mu \mathrm{s}$, which is close enough to $37.5 \mu \mathrm{s}(2 \sigma=150 \mu \mathrm{s})$ considered in this work. Following previous studies (Gjesteland et al., 2010; Østgaard et al., 2012), the temporal distribution of source photons is assumed to be Gaussian-shaped and the duration is assumed to be $150 \mu$ s between $\pm 2 \sigma$. The shape of the photon pulse at the spacecraft altitude also highly depends on Compton scattering effects. The contribution of those effects for the same source, of course, will be different for various source to spacecraft nadir horizontal distances and source altitudes due to differences in how the gamma-rays propagate. As we mentioned previously, individual TGFs have a low number of counts, which makes subsequent analysis of such events challenging. Pulse pileup due to more than one photon hitting the detector within a short period of time is an additional challenge. The pulse pileup effects can be taken into account and corrections can be made for the occurring spectral distortions (Chaplin et al., 2013). The pulse pileup code developed by Chaplin et al. (2013) takes an input spectrum and then outputs the corrected spectrum for the assumed incident photon rate. In Mailyan et al. (2016), the pulse pileup corrections were made assuming a constant photon rate. However, the rates observed with space instruments tend to approximate Gaussian or lognormal time profiles (Fitzpatrick et al., 2014; Foley et al., 2014) and are likely good approximations of the time histories of photons arriving to the detectors on Fermi. By assuming a constant photon rate, the photon temporal distribution was approximated as a step function, which is a compromise while dealing with a low number of measured photon counts. In order to determine whether such an approximation is justified, we fit the brightest GBM TGFs to typical 100-MV lightning leader models at four different altitudes of 10, 12, 15 , and $20 \mathrm{~km}$. Figure 3 shows an example of the temporal distribution of source photons produced by a 100-MV lightning leader, fitted by a Gaussian. Two versions of corrections for pulse pileup were considered for the same set of TGFs. In the first version, the standard constant-rate correction was applied and in the other, a time-resolved pulse pileup correction was done. To perform the time-resolved pulse pileup correction, the model photon pulse (for energies $>200 \mathrm{keV}$ ) at the spacecraft altitude was fitted by a Gaussian. For each such fit (for each model used) the $1 \sigma$ region was calculated, and for photons in that region we assumed a photon rate double that of the rise and tail parts. The model photons and observed counts were sorted in the same manner. Seventy percent of counts at the peak (approximate $1 \sigma$ region) and the rest of the counts at the initial and tail parts of the TGF were separated. Logarithmic likelihood values were calculated for the high-rate ( $1 \sigma$ region) and low-rate
Figure 3. The time distribution of a 15-km altitude 100-MV lightning leader model fitted by a Gaussian. The vertical lines are to indicate the $1 \sigma$ region. 

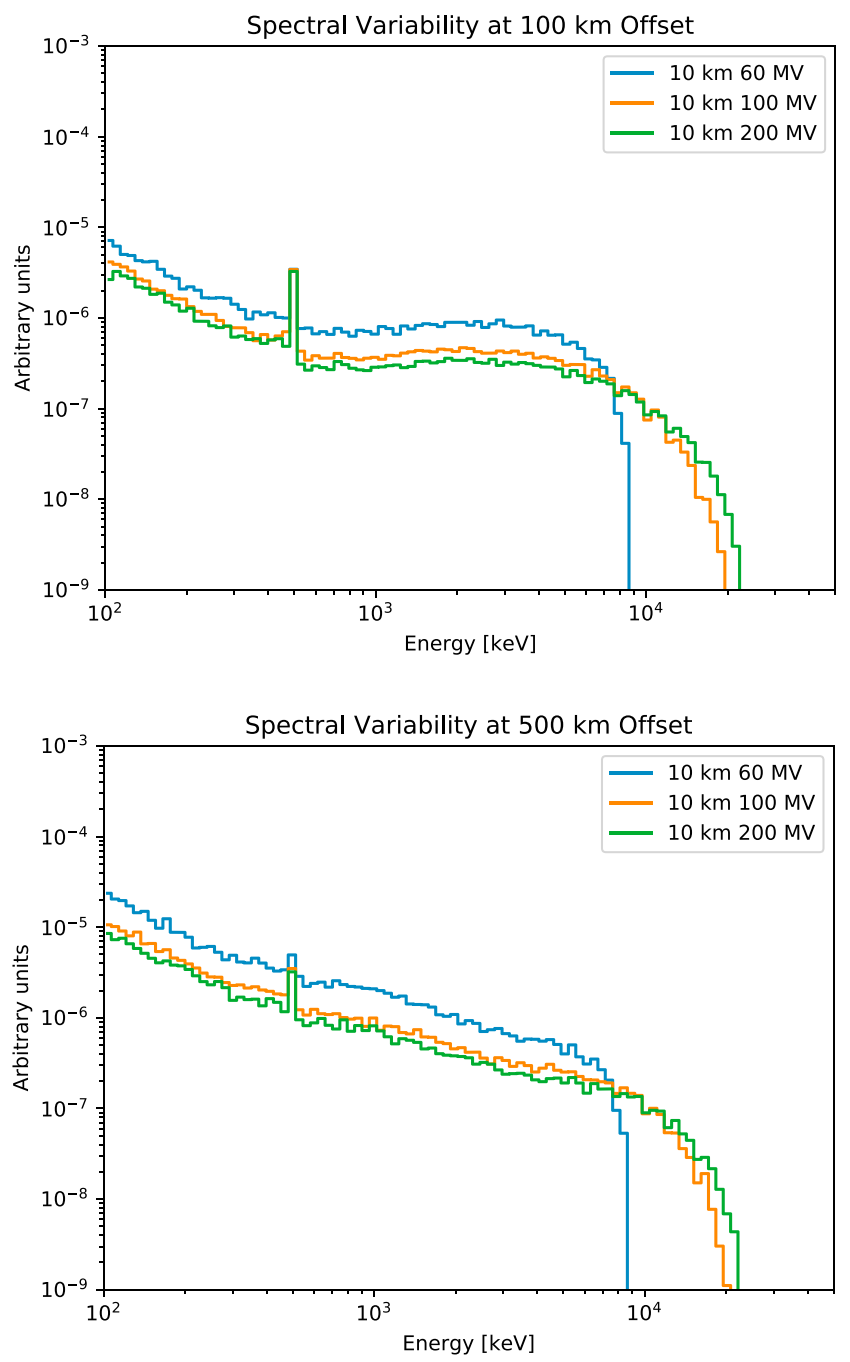

Figure 4. The differential energy spectra of 10-km altitude lightning leader models with 60-, 100-, and 200-MV leader potentials at 100- (top panel) and 500-km (bottom panel) offset distances. (rise and tail) parts separately then summed for those high-rate and low-rate parts. We compared the likelihood analysis results for the constant and variable photon rate calculations and could not find differences in the conclusions. The best fit models and the second, the third, and so on preferred models ( -2 times logarithmic likelihood values differ from the best fit model by less than five as in Mailyan et al. (2016) analysis) were the same for both analyses. As there are only a limited number of counts for each TGF, the constant-rate assumption is found to be a good approximation. Thus, in the rest of the calculations in this paper, we will use the simplified constant rate assumption while making corrections for the pulse pileup effects. At the same time, it is worth mentioning that the photon rates obtained by the time-averaged individual TGF spectral fits give slightly (about 20\%) lower photon rates, than would get by considering time-resolved fits. However, we do not see any indication of "super-TGFs" with very high photon rates at the peak.

\section{Results}

\subsection{Spectral Dependence}

In the previous analyses of GBM-measured TGF spectra (e.g., Fitzpatrick et al., 2014; Mailyan et al., 2016), while fitting cumulative or individual TGF spectra, only one electric field value of $400 \mathrm{kV} / \mathrm{m}$ was considered. This value is a standard for modeling RREA process in the large-scale atmospheric electric fields. Similarly, a $100-\mathrm{MV}$ potential drop is considered to be a typical value of potential drop used for modeling the electron acceleration at the tip of lightning leaders resulting in a TGF. Until recently, there was an opinion that the spectrum of the accelerated electrons in fully developed RREAs should not depend on the electric field, and the electron spectrum can be described by an exponential function with a cutoff at 7.3 MeV (see, e.g., Dwyer \& Babich, 2011). However, Cramer et al. (2017) showed that the accelerated electron spectrum significantly depends on the electric field strength near the RREA threshold, even for homogeneous electric fields. Consequently, photon spectra produced by different large-scale homogeneous electric fields can differ. An intrinsic dependence of photon spectra is also predicted in the lightning-leader-based TGF production model as a function of the potential drop as RREAs only partially develop if the potential drop is not high enough (Celestin et al., 2015). Fully developed RREAs can be seen as a limit case of the leader model as the potential drop increases. We are able to study those spectral variability effects by fitting GBM TGF data with lightning leader models with potentials of 60, 100, and $200 \mathrm{MV}$. In the top and bottom panels of Figure 4, the differential energy spectra of modeled photons at the spacecraft altitude are shown for 100- and 500-km source offset distances, respectively. At both distances, the spectral hardening due to the increasing leader potential is obvious. At $500 \mathrm{~km}$, we can also see the spectral softening due to Compton scattering effects. The 60-MV leader, for example, provides a softer spectra, which may explain some GBM observations of soft TGFs. On the other hand, there is a slight spectral hardening observed for the 200-MV model in comparison with the standard 100-MV leader model. This spectral dependence is promising for obtaining improved fits of the GBM-observed data (Roberts et al., 2018). In the following, we will consider two cases of observed TGFs with soft and hard spectra.

\subsection{Soft TGFs at Large Offset Distances: Tilted Photon Beams}

In the data set analyzed by Mailyan et al. (2016), there were a few very soft events, which were observed at large offset distances. For those cases, the observed soft spectra could be explained using the 20-km altitude RREA models, which allow low-energy photons to escape atmospheric attenuation. Narrower beams produced by RREA mechanisms in a large-scale atmospheric electric field allowed for a larger contribution of Compton-scattered lower-energy particles observed at large offset distances. In addition, no significant pulse pileup effects were estimated for those large-offset TGFs, which makes the analysis of those events 


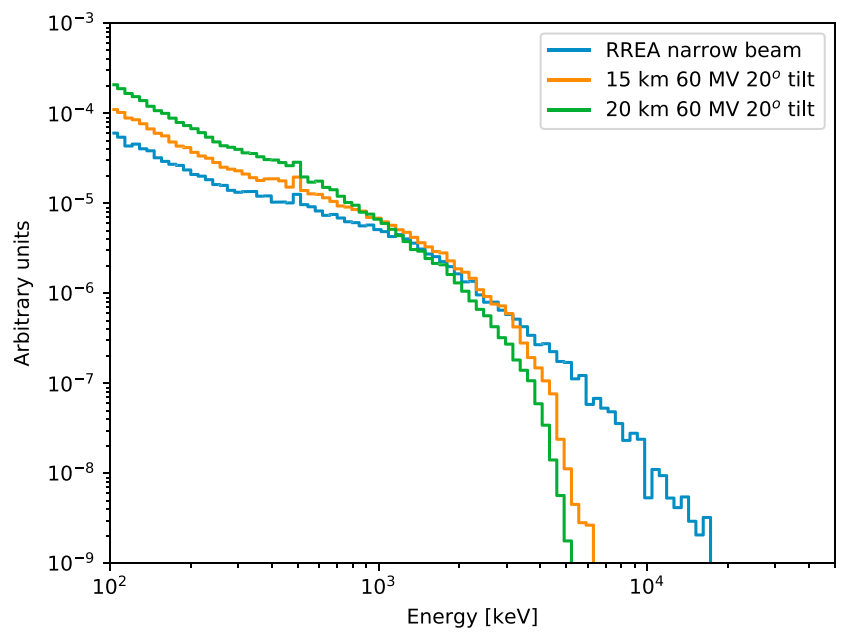

Figure 5. The modeled energy spectra of TGF120120412 at 475-km offset distance. RREA narrow 20-km model along with 20-km 60-MV lightning leader and $15-\mathrm{km} 60-\mathrm{MV}$ models with $20^{\circ}$ tilt. TGF $=$ terrestrial gamma-ray flash; RREA = Relativistic Runaway Electron Avalanche. simpler as we do not have to apply the pulse pilup corrections. Based on these few events, which were best fit by narrow beam models, Mailyan et al. (2016) concluded that for those cases, large-scale RREA model works better since wider beams (supposedly produced by lightning leader model) could be rejected with a high level of confidence. At the same time, it was mentioned that a wider beam tilted opposite to the spacecraft direction would provide more Compton scattered photons and consequently softer spectra at larger offset distances from the source.

In Figure 5, the differential energy spectra of the photons at 475-km offset distance corresponding to the soft event TGF120120412 are shown. As we can see, the tilted beam of $20^{\circ}$ from a 60 -MV leader already is softer than the large-scale RREA spectrum. The $60-\mathrm{MV}$ model with a $40^{\circ}$ tilt can make even softer spectra, which is too soft to match the data. As a result, lower-altitude tilted beam models can provide better fits to the data, by making the spectra a bit harder due to the atmospheric attenuation of the low-energy gamma rays. For this particular TGF, 15-km 60-MV model with a tilt of $20^{\circ}$ appears to be the best fit. Figure 5 presents the $20-\mathrm{km}$ altitude with $20^{\circ}$ tilt $60-\mathrm{MV}$ leader model (which was too soft to fit) and the best fit $15-\mathrm{km}$ altitude $60-\mathrm{MV}$ leader $20^{\circ}$ tilted beam model.

Figure 6 shows the energy spectra of the TGF120120412 along with the best fit lightning leader model (top) and the best fit RREA model (bottom). Upper limits corresponding to $2 \sigma$ are calculated from the model and indicated by arrows, when no photon counts were observed in a particular histogram bin. As we can see, the energy spectrum of TGF120120412 can be perfectly fitted using the lightning leader model with a tilt angle of $20^{\circ}$, a leader potential of $60 \mathrm{MV}$, and a source altitude of $15 \mathrm{~km}$. It is worth mentioning that the best fit RREA model is not tilted and electric field variability of the RREA spectrum is not considered in this work, nor in Mailyan et al. (2016).
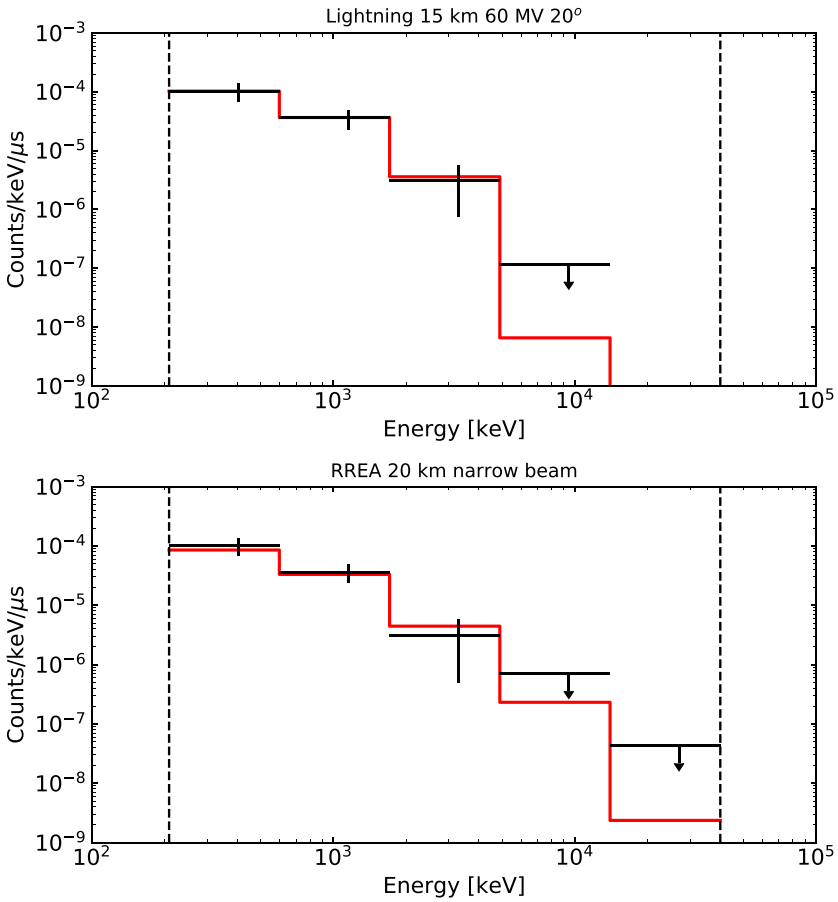

Figure 6. The modeled and measured energy spectra of TGF120120412 at 475-km offset distance. RREA narrow 20-km model (bottom plot) and 15-km 60-MV lightning leader models with a $20^{\circ}$ tilt opposite to the spacecraft (top plot). TGF $=$ terrestrial gamma-ray flash; RREA = Relativistic Runaway Electron Avalanche. 

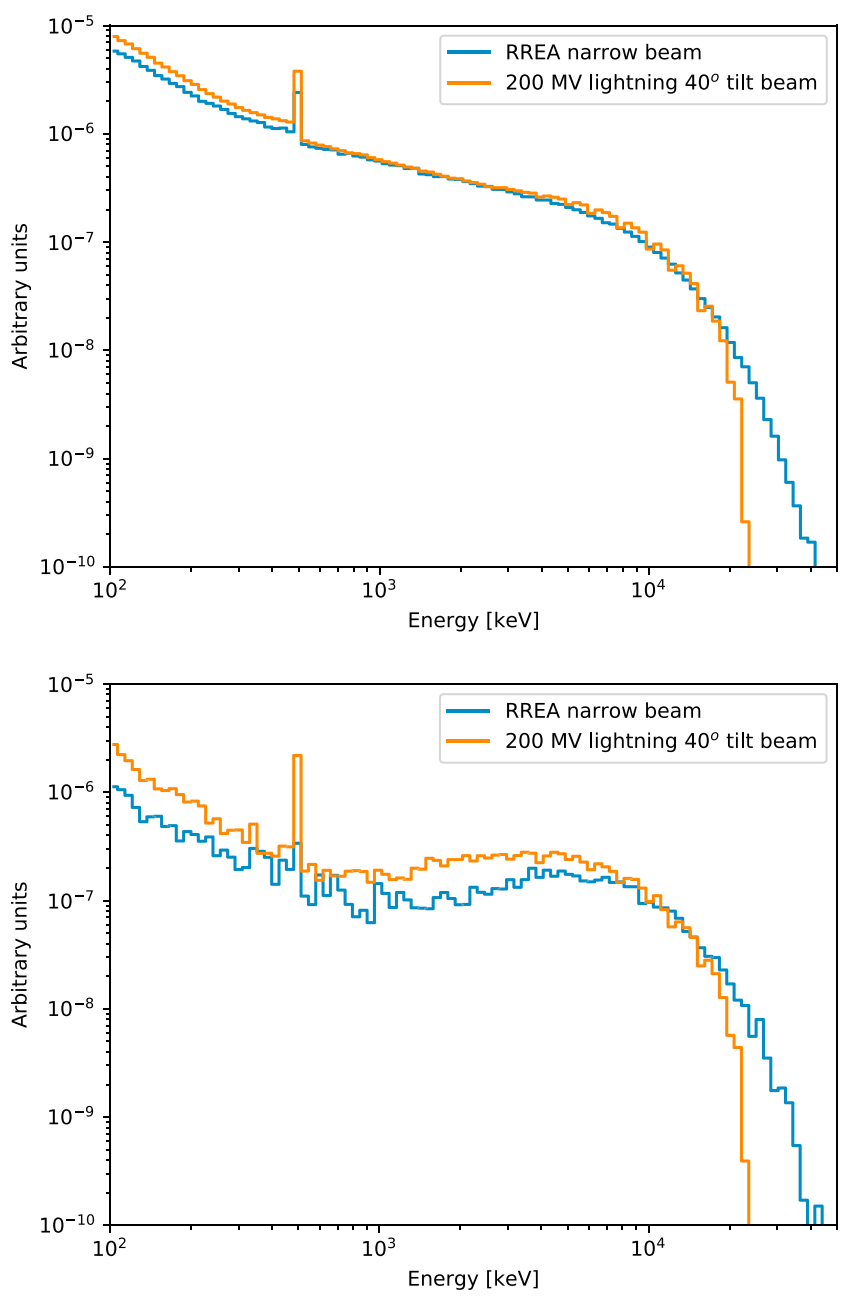

Figure 7. The modeled differential energy spectra of TGF100909539 observed at 102-km offset distance. RREA narrow $10-\mathrm{km}$ model along with lightning leader $10-\mathrm{km}$ 200-MV model with $40^{\circ}$ tilt toward the detector for all photons (top plot) and photons at 102-km offset distance only (bottom plot).

\subsection{Hard TGFs at Small Offset Distances}

TGF100909539 analyzed in Mailyan et al. (2016), is a perfect example of a hard TGF with photon counts above $20 \mathrm{MeV}$ and almost no counts below $1 \mathrm{MeV}$, observed at 102-km source/spacecraft nadir offset distance. From the considered RREA models, the deep source altitude could explain the absence of the low-energy component and narrow beams of the RREA photons could provide higher-energy particles at the spacecraft altitude with less contribution of Compton scattering effects.

We examined whether or not lightning leader models can provide better fits to this particular TGF. In Figure 7, the simulated lightning leader spectrum is presented along with the RREA spectrum. Both spectra fit the TGF data well, with the data being best fit using a model with a 200-MV leader potential tilted $40^{\circ}$ toward the spacecraft from a source altitude of $10 \mathrm{~km}$. In the top of Figure 7, comparisons of the best lightning leader and best fit RREA narrow beam 10-km altitude models are shown for photons at all offset distances. The highest photon energy of 200-MV lightning leader model spectrum is significantly less than the highest photon energy in the RREA model. In the bottom panel, a similar plot shows the difference between lightning leader and RREA models at 102-km offset distance (within 10-km ring around this offset) where the TGF was actually observed. The maximum energy of the RREA spectrum is 40-MeV, which is twice as high as the maximum energy predicted using the lightning leader spectral model (20 MeV). Note that a spectrum typical of RREA in homogeneous fields can be obtained for the lightning leader models assuming a higher potential drop of $300 \mathrm{MV}$ (Celestin et al., 2015). 

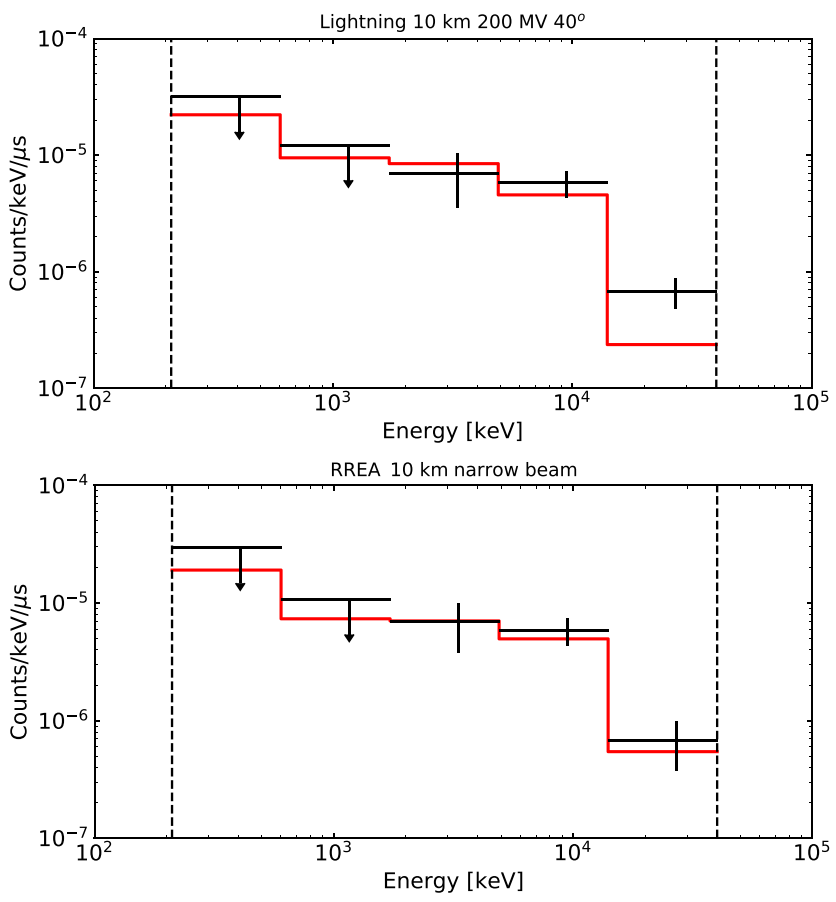

Figure 8. The modeled and measured differential energy spectra of TGF100909539 observed at 102-km offset distance. RREA narrow 10-km model (bottom plot) along with lightning leader 10-km 200-MV model with $40^{\circ}$ tilt toward the detector (top plot) and observed TGF data at 102-km offset distance.

In Figure 8, the observed TGF100909539 spectrum along with the best fit lightning leader (top) and RREA (bottom) models are shown. Both models match the observed spectrum fairly well except for the highest energy bin above $10 \mathrm{MeV}$. While the best fit lightning leader model shows a deficit of particles at these energies, the RREA model provides sufficient photons above $10 \mathrm{MeV}$ to fit adequately the observations within the error bars.

Thus, using lightning leader models with various leader potentials, source altitudes and using tilt beams help improve the best fits obtained with RREA models, however, some hard TGFs were not better fit with lightning leader models for potential drops $\sim 200 \mathrm{MV}$.

\section{Discussion and Conclusions}

In this paper we have analyzed a data set of 66 TGFs observed by Fermi GBM, performing spectral analysis for each TGF. We found there are cases where the fits by the RREA model presented by Mailyan et al. (2016) can be significantly improved using lightning leader models. Also, there are events, which are still better fit by models relying on fully developed RREA due to its harder spectrum. The electrons of some TGFs may be predominantly accelerated in large-scale homogeneous electric fields by the RREA mechanism, while for other TGFs, the most of the acceleration may take place in the inhomogeneous electric fields of lightning leaders.

In the data set considered in this work, 39 TGFs (out of 66 TGFs) can be best fit with fully developed RREA models, while 27 events can be best fit using the lightning leader models. At the same time, in most of the cases, we cannot favor one mechanism over the other based on the likelihood analysis with a high level of confidence. From lightning leader models, 200-MV leader models with and without a tilt toward the spacecraft are preferred as they provide harder spectra. There are only four events were RREA models only were preferred (over lightning leader models) and all of them had hard energy spectra with photon counts above $20 \mathrm{MeV}$. It is worth reminding that tilted beams were not considered for the RREA models, and in this work we primarily focus on the leader models. From the 39 events best fit by RREA models, 24 were best fit by narrow models and 15 were best fit by wide RREA models. From 27 TGFs best fit by lightning leader models, 17 were best fit by 200-MV models, 8 were best fit by $100 \mathrm{MV}$, and only 2 TGFs were best 


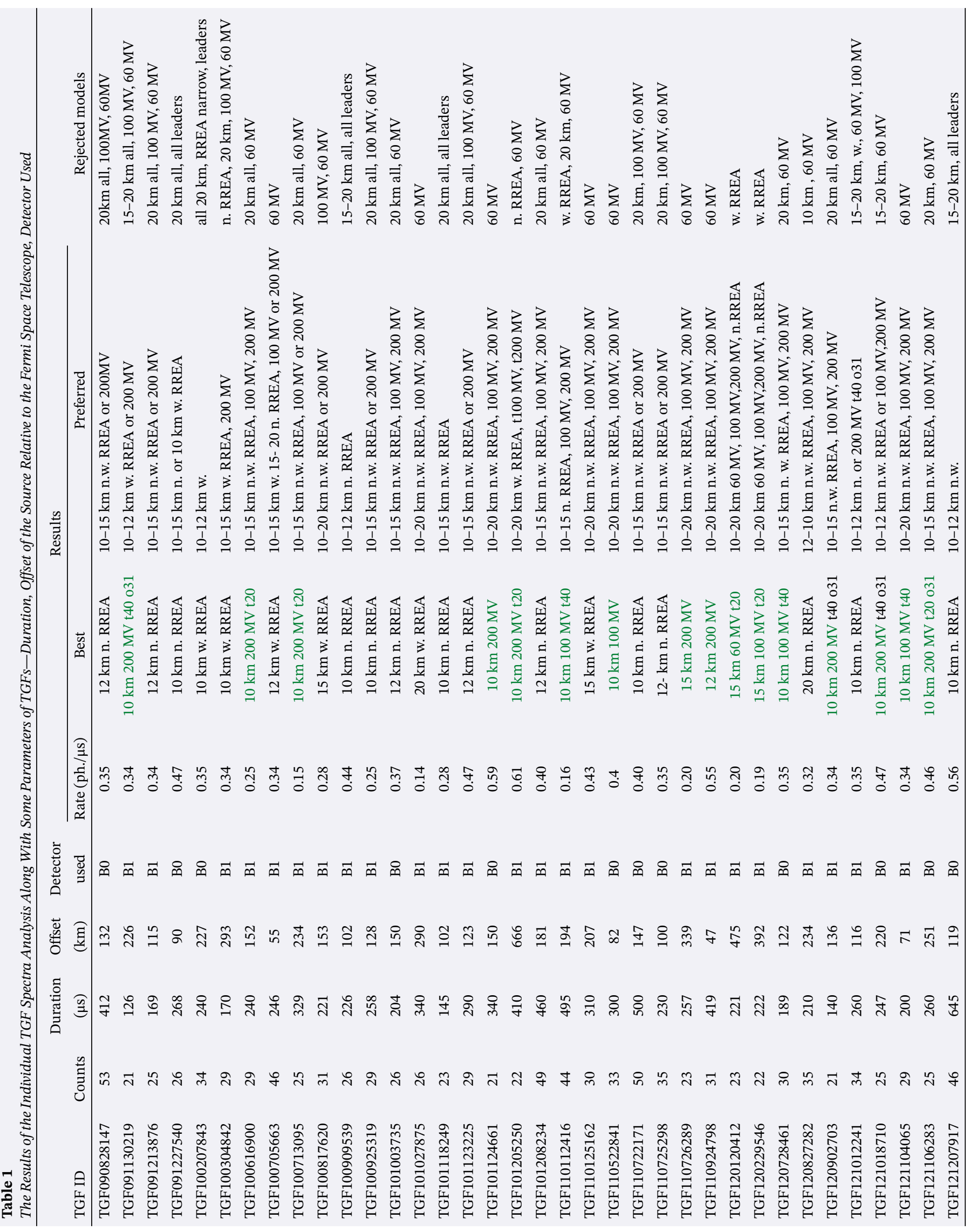




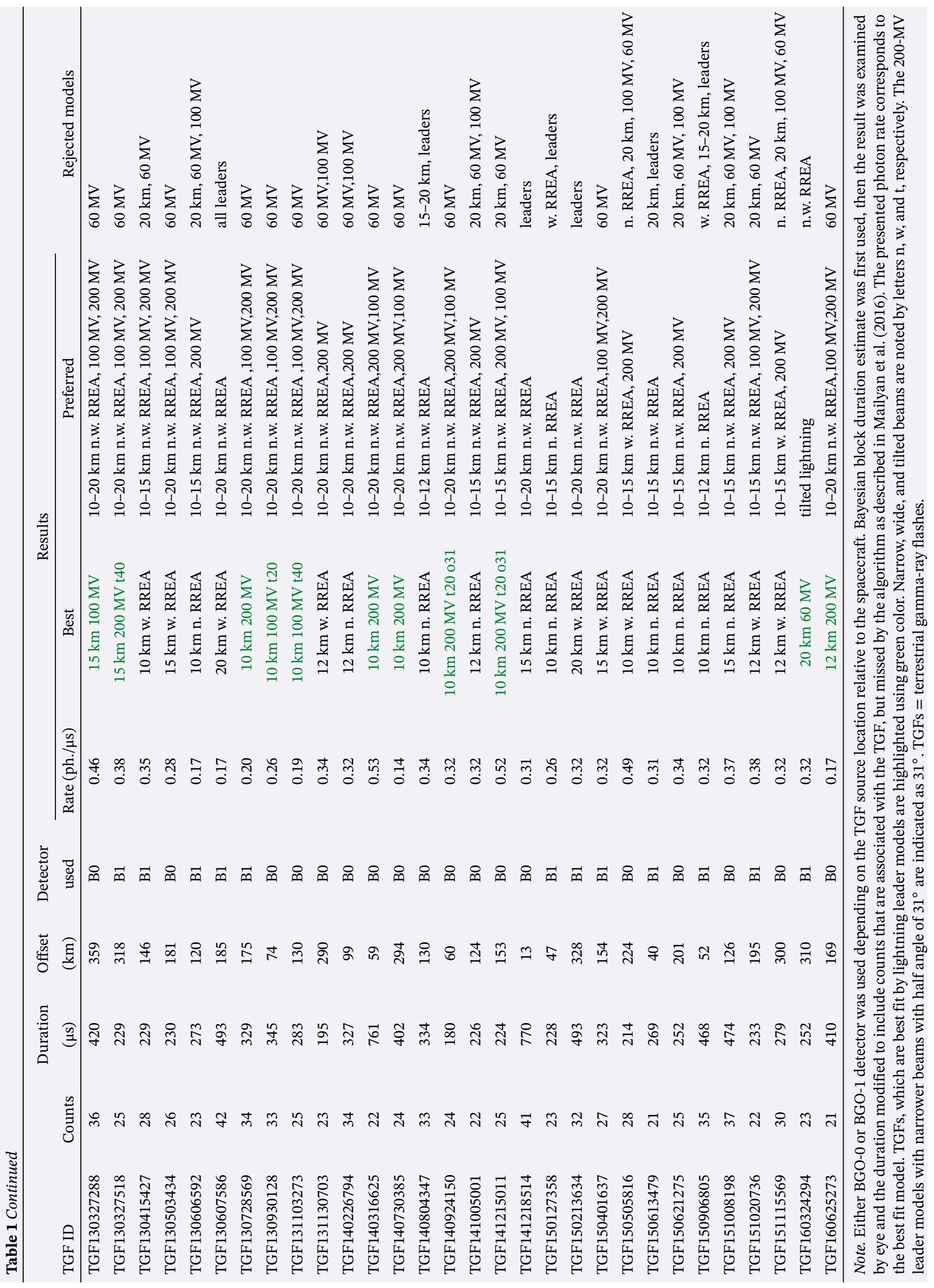




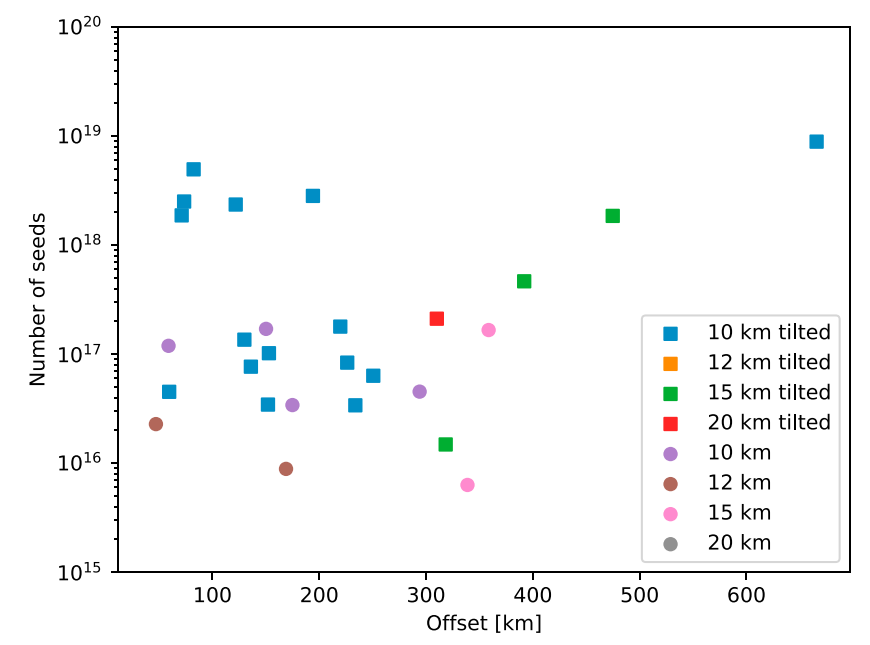

Figure 9. Total number of seed electrons with energies greater than $10 \mathrm{keV}$ versus subsatellite offset distance of different TGF events measured by Fermi GBM. The results are obtained using the best fit lightning models. The production altitude of TGF source is indicated by different colors. Most of the best fit models are tilted. TGF $=$ terrestrial gamma-ray flash; GBM $=$ Gamma-ray Burst Monitor. fit by a 60 -MV model. In general, low-altitude models were preferred for both lightning leader and RREA models. Out of 66 TGFs, 36 could be best fit by a $10-\mathrm{km}$ altitude model, 14 events were best fit by a $12-\mathrm{km}$ model, and 11 events by a $15-\mathrm{km}$ model. Only five 5 events were best fit by a $20-\mathrm{km}$ model. Most of the time all 20-km models and 60-MV lightning leader models can be rejected with a high level of confidence as the GBM-observed spectra tend to be hard. However, due to the spectral diversity of the data set analyzed in this paper (events occurring in 2013-2016), there are exceptions. For example, high-altitude, low leader potential models can also best fit some observed TGF data. TGF160324294 is the only event for which RREA models considered in this work could be rejected. This is a very soft TGF with only one count above $1 \mathrm{MeV}$ and can be best fit by a tilted 60-MV leader, 20-km source altitude model. All the events analyzed in this paper are presented in Table 1, along with their best, preferred, and rejected models.

A critical parameter in TGF studies is the source brightness, that is, total number of seed electrons and/or photons, a parameter directly related to the production mechanism and subsequent avalanche processes. In the present paper, using satellite-measured photon fluence and corresponding best fit leader model, we have estimated this number in three steps. First, we derive the true fluxes of gamma-rays incident on Fermi detectors by taking the instrumental effects into account, for example, pulse pileup, dead time, and effective detection area. Second, we calculate the number of source bremsstrahlung photons that is required at the production altitude in order to reproduce the gamma-ray flux obtained in the first step. Finally, this number of source photons is converted into seed electrons using a conversion factor. Specifically, this factor describes the total number of bremsstrahlung photons $(>10 \mathrm{keV})$ produced per electron injected in Monte Carlo simulations and is calculated explicitly following the procedure described in Celestin et al. (2015). In the lightning leader model, it is mostly controlled by the potential drop formed in the leader tip region, being almost invariant of production altitude. The factor is approximately 3.4, 6.3, and 59.7 for leader potentials of 60, 100, and $200 \mathrm{MV}$, respectively, increasing almost exponentially in the potential range above $60 \mathrm{MV}$.

Figure 9 shows the total number of seed electrons with energies greater than $10 \mathrm{keV}$ versus subsatellite offset distance of different TGF events measured by Fermi GBM. The results are obtained using the corresponding best fit lightning leader parameters. A minimum energy of $10 \mathrm{keV}$ is used as that is the lowest energy consistently used in the present Monte Carlo simulations of electrons and photons. Counting the total number of electrons that are truly runaway in inhomogeneous electric fields is complicated since it requires comparing the energy of each electron with the electric field at the position of this electron. The total number of source electrons (with energies greater than $10 \mathrm{keV}$ ) is found to be roughly in the range between $6 \times 10^{15}$ and $9 \times 10^{18}$, with a majority between $1 \times 10^{16}$ and $1 \times 10^{17}$. If we take the avalanche multiplication into account, most estimated numbers of seeds are consistent with previously reported estimations using measurements of radio signals and gamma-ray fluxes (e.g., Cummer et al., 2015; Dwyer et al., 2017). Note that this number is generally higher than the theoretical value suggested by Celestin et al. (2015), which is derived using ground-based measurements of X-ray bursts (Schaal et al., 2012) by approximately 1 order of magnitude. This is possibly due to the fact that the subset of TGFs used in this study, that is, TGFs for which individual spectra can be estimated, corresponds to the highest part of the fluence distribution. We have checked in a separate test that, if the screening effects are not considered (Lehtinen, 2000), or if the Seltzer-Berger cross section (Seltzer \& Berger, 1986) is instead used, the conversion factor (number of photons with energies greater than $10 \mathrm{keV}$ produced per electron injected) could change, but by less than 1 order of magnitude. It is, thus, important to note that different cross sections of bremsstrahlung radiation may somewhat change this estimation, that is, by less than 1 order of magnitude. It can be seen from Figure 9 that most of the best fit source altitudes are below $15 \mathrm{~km}$, in good agreement with recent radio measurements that TGFs are correlated with initial leader steps during the initial development stages of intracloud discharges (Marshall et al., 2013). Interestingly, most of the deep TGF sources, for example, at 10 and $12 \mathrm{~km}$, can best explain GBM measurements at nadir-offset distances less than $300 \mathrm{~km}$. This is because source bremsstrahlung photons 
originating from lower altitudes would experience more attenuation in the atmosphere, and thus less likely to be registered as a bright event by Fermi GBM at offset distances larger than $300 \mathrm{~km}$. The 20-km source (red point in Figure 9) is related to a low leader potential of $60 \mathrm{MV}$ and tilted source beam. Also, at the same offset distance with the same production altitude of $10 \mathrm{~km}$ (blue points in Figure 9), the number of seeds can change by two orders of magnitude, which indicates the variation of the leader potential and the fact that the source photon beam is tilted.

In summary, it is difficult in most cases to convincingly prove or reject either the lightning leader or fully developed RREA models. Multipoint measurements of TGFs would allow to put more strict constrains on the photon beaming geometry by having measurements available at various offset distances from the source. However, it should be pointed out that both processes may occur in thundercloud electric fields. Leader-accelerated electrons can also runaway into uniform, large-scale thunderstorm fields, resulting in narrower beams. Thus, beaming only cannot automatically be used to constrain the TGF production mechanisms. Additional broadband radio, optical and radar measurements would be helpful for future TGF analyses.

\section{Acknowledgments}

The Fermi GBM Collaboration acknowledges the support of NASA in the United States and DRL in Germany. We thank NASA for support from Fermi Guest Investigation Grant 80NSSC17K0514. Sebastien Celestin's work was supported by the French space agency (CNES) in the framework of the satellite project TARANIS. Joesph Dwyer acknowledges the support from Air Force Office of Scientific Research under Award FA9550-16-1-0396. The authors wish to thank the World Wide Lightning Location Network (http:// wwlln.net), a collaboration among over 50 universities and institutions, for providing the lightning location data used in this paper. We also acknowledge Earth Networks for providing the ENTLN data examined in this study. We also acknowledge Earth Networks for providing the Earth Networks Total Lightning Network (ENTLN) (https://www. earthnetworks.com/why-us/networks/ lightning/) data used in this study. Researchers may contact customercare@earthnetworks.com to arrange access to ENTLN data. The authors are grateful to Melissa Gibby for her help during the preparation of this paper. All GBM data used in this paper are available at http://fermi.gsfc. nasa.gov/ssc/data/access/gbm/. DRMs for the TGFs analyzed in this paper are available at http://gammaray.nsstc. nasa.gov/gbm/publications/mailyan_ rspfiles/rsp_desc.html. Requests for the simulations code can be made to the corresponding author. A catalog describing TGF parameters is available at http://fermi.gsfc.nasa.gov/ssc/data/ access/gbm/tgf/. The data used in figures and results of this paper are available at https://github.com/ bagratmailyan/tgfspectra.

\section{References}

Balanis, C. A. (1989). Advanced engineering electromagnetics. New York: John Wiley.

Bazelyan, E. M., \& Raizer, Y. P. (2000). Lightning physics and lightning protection. Bristol, PA: Instit. of Phys.

Briggs, M., Fishman, G., Connaughton, V., Bhat, P. N., Paciesas, W., Preece, R., et al. (2010). First results on terrestrial gamma ray flashes from the Fermi gamma-ray burst monitor. Journal of Geophysical Research, 115, A07323. https://doi.org/10.1029/2009JA015242

Briggs, M. S., Xiong, S., Connaughton, V., Tierney, D., Fitzpatrick, G., Foley, S., et al. (2013). Terrestrial gamma-ray flashes in the Fermi era: Improved observations and analysis methods. Journal of Geophysical Research: Space Physics, 118, 3805-3830. https://doi.org/10.1002/ jgra.50205

Carlson, B., Lehtinen, N. G., \& Inan, U. S. (2007). Constraints on terrestrial gamma ray flash production from satellite observation. Geophysical Research Letters, 34, L08809. https://doi.org/10.1029/2006GL029229

Celestin, S., \& Pasko, V. P. (2010). Soft collisions in relativistic runaway electron avalanches. Journal of Physics D: Applied Physics, 43, 315206. https://doi.org/10.1088/0022-3727/43/31/315206

Celestin, S., \& Pasko, V. P. (2011). Energy and fluxes of thermal runaway electrons produced by exponential growth of streamers during the stepping of lightning leaders and in transient luminous events. Journal of Geophysical Research, 116, A03315. https://doi.org/10.1029/ 2010JA016260

Celestin, S., Xu, W., \& Pasko, V. P. (2012). Terrestrial gamma ray flashes with energies up to $100 \mathrm{MeV}$ produced by nonequilibrium acceleration of electrons in lightning. Journal of Geophysical Research, 117, A05315. https://doi.org/10.1029/2012JA017535

Celestin, S., Xu, W., \& Pasko, V. P. (2015). Variability in fluence and spectrum of high-energy photon bursts produced by lightning leaders. Journal of Geophysical Research: Space Physics, 120, 10,712-10,723. https://doi.org/10.1002/2015JA021410

Chaplin, V., Bhat, N. P., Briggs, M. S., \& Connaughton, V. (2013). Analytical modeling of pulse-pileup distortion using the true pulse shape: Applications to Fermi-GBM. Nuclear Instruments and Methods in Physics Research Section A: Accelerators, Spectrometers, Detectors and Associated Equipment, 717, 21-36.

Cramer, E. S., Mailyan, B. G., Célestin, S., \& Dwyer, J. R. (2017). A simulation study on the electric field spectral dependence of thunderstorm ground enhancements and gamma ray glows. Journal of Geophysical Research: Atmospheres, 122, 4763-4772. https://doi.org/10. 1002/2016JD026422

Cummer, S. A., Lyu, F., Briggs, M. S., Fitzpatrick, G., Roberts, O. J., \& Dwyer, J. R. (2015). Lightning leader altitude progression in terrestrial gamma-ray flashes. Geophysical Research Letters, 42, 7792-7798. https://doi.org/10.1002/2015GL065228

Dwyer, J. (2003). A fundamental limit on electric fields in air. Geophysical Research Letters, 30(20), 2055. https://doi.org/10.1029/ 2003GL017781

Dwyer, J. R. (2008). Source mechanisms of terrestrial gamma-ray flashes. Journal of Geophysical Research, 113, D10103. https://doi.org/10. 1029/2007JD009248

Dwyer, J. R., \& Babich, L. P. (2011). Low-energy electron production by relativistic runaway electron avalanches in air. Journal of Geophysical Research, 116, A09301. https://doi.org/10.1029/2011JA016494

Dwyer, J. R., Liu, N., Grove, J. E., Rassoul, H., \& Smith, D. M. (2017). Characterizing the source properties of terrestrial gamma ray flashes. Journal of Geophysical Research: Space Physics, 122, 8915-8932. https://doi.org/10.1002/2017JA024141

Dwyer, J., \& Smith, D. (2005). A comparison between Monte Carlo simulations of runaway breakdown and terrestrial gamma-ray flash observations. Geophysical Research Letters, 32, L22804. https://doi.org/10.1029/2005GL023848

Dwyer, J. R., Smith, D. M., \& Cummer, S. A. (2012). High-energy atmospheric physics: Terrestrial gamma-ray flashes and related phenomena. Space Science Review, 173, 133-196. https://doi.org/10.1007/s11214-012-9894-0

Fishman, G. J., Bhat, P. N., Mallozzi, R., Horack, J. M., Koshut, T., Kouveliotou, C., et al. (1994). Discovery of intense gamma-ray flashes of atmospheric origin. Science, 59, 1313-1316.

Fitzpatrick, G., Cramer, E., McBreen, S., Briggs, M. S., Foley, S., Tierney, D., et al. (2014). Compton scattering in terrestrial gamma-ray flashes detected with the Fermi gamma-ray burst monitor. Physical Review D, 90(4), 43008.

Foley, S., Fitzpatrick, G., Briggs, M. S., Connaughton, V., Tierney, D., McBreen, S., et al. (2014). Pulse properties of terrestrial gamma-ray flashes detected by the Fermi gamma-ray burst monitor. Journal of Geophysical Research: Space Physics, 119, 5931-5942. https://doi.org/ 10.1002/2014JA019805

Gjesteland, T., Østgaard, N., Connell, P., Stadsnes, J., \& Fishman, G. (2010). Effects of dead time losses on terrestrial gamma ray flash measurements with the burst and transient source experiment. Journal of Geophysical Research, 115, A00E21. https://doi.org/10.1029/ 2009JA014578 
Gjesteland, T., Østgaard, N., Laviola, S., Miglietta, M., Arnone, E., Marisaldi, M., et al. (2015). Observation of intrinsically bright terrestrial gamma ray flashes from the Mediterranean basin. Journal of Geophysical Research: Atmospheres, 120, 12,143-12,156. https://doi.org/10. 1002/2015jd023704

Hutchins, M., Holzworth, R., Brundell, J., \& Rodger, C. (2012). Relative detection efficiency of the world wide lightning location network. Radio Science, 47, RS6005. https://doi.org/10.1029/2012RS005049

Lehtinen, N. G (2000). Relativistic runaway electrons above thunderstorms (Ph.D. thesis), stanford university Ph. D. dissertation.

Lyu, F. c., Cummer, S. A., Lu, G. p., Zhou, X., \& Weinert, J. (2016). Imaging lightning intracloud initial stepped leaders by low-frequency interferometric lightning mapping array. Geophysical Research Letters, 43, 5516-5523. https://doi.org/10.1002/2016GL069267

Mailyan, B. G., Briggs, M. S., Cramer, E. S., Fitzpatrick, G., Roberts, O. J., Stanbro, M., et al. (2016). The spectroscopy of individual terrestrial gamma-ray flashes: Constraining the source properties. Journal of Geophysical Research: Space Physics, 121, 11,346-11,363. https://doi. org/10.1002/2016JA022702

Mallios, S. A., Celestin, S., \& Pasko, V. P. (2013). Production of very high potential differences by intracloud lightning discharges in connection with terrestrial gamma ray flashes. Journal of Geophysical Research: Space Physics, 118, 912-918. https://doi.org/10.1002/jgra 50109

Marisaldi, M., Fuschino, F., Labanti, C., Galli, M., Longo, F., Del Monte, E., et al. (2010). Detection of terrestrial gamma ray flashes up to $40 \mathrm{MeV}$ by the AGILE satellite. Journal of Geophysical Research, 115, A00E13. https://doi.org/10.1029/2009JA014502

Marshall, T., Stolzenburg, M., Karunarathne, S., Cummer, S., Lu, G., Betz, H.-D., et al. (2013). Initial breakdown pulses in intracloud lightning flashes and their relation to terrestrial gamma ray flashes. Journal of Geophysical Research: Atmospheres, 118, 10,907-10,925. https://doi.org/10.1002/jgrd.50866

Meegan, C., Lichti, G., Bhat, P. N., Bissaldi, E., Briggs, M. S., Connaughton, V., et al. (2009). The Fermi gamma-ray burst monitor. The Astrophysical Journal, 702(1), 791.

Moss, G. D., Pasko, V. P., Liu, N., \& Veronis, G. (2006). Monte Carlo model for analysis of thermal runaway electrons in streamer tips in transient luminous events and streamer zones of lightning leaders. Journal of Geophysical Research, 111, A02307. https://doi.org/10. 1029/2005JA011350

Østgaard, N., Gjesteland, T., Hansen, R., Collier, A., \& Carlson, B. (2012). The true fluence distribution of terrestrial gamma flashes at satellite altitude. Journal of Geophysical Research, 117, A03327. https://doi.org/10.1029/2011JA017365

Østgaard, N., Gjesteland, T., Stadsnes, J., Connell, P. H., \& Carlson, B. (2008). Production altitude and time delays of the terrestrial gamma flashes: Revisiting the Burst and Transient Source Experiment spectra. Journal of Geophysical Research, 113, A02307. https://doi.org/10. 1029/2007JA012618

Rakov, V. A., \& Uman, M. A. (2003). Lightning: Physics and effects. Cambridge, U. K: Cambridge Univ. Press.

Roberts, O., Fitzpatrick, G., Stanbro, M., McBreen, S., Briggs, M., Holzworth, R., et al. (2018). The first Fermi-GBM terrestrial gamma ray flash catalog. Journal of Geophysical Research: Space Physics, 123, 4381-4401. https://doi.org/10.1029/2017JA024837

Schaal, M. M., Dwyer, J. R., Saleh, Z. H., Rassoul, H. K., Hill, J. D., Jordan, D. M., \& Uman, M. A. (2012). Spatial and energy distributions of X-ray emissions from leaders in natural and rocket triggered lightning. Journal of Geophysical Research, 117, D15201. https://doi.org/ 10.1029/2012JD017897

Seltzer, S. M., \& Berger, M. J. (1986). Bremsstrahlung energy spectra from electrons with kinetic energy $1 \mathrm{keV}-10 \mathrm{GeV}$ incident on screened nuclei and orbital electrons of neutral atoms with $\mathrm{z}=1-100$. Atomic data and nuclear data tables, 35(3), 345-418.

Skeltved, A. B., Østgaard, N., Mezentsev, A., Lehtinen, N., \& Carlson, B. (2017). Constraints to do realistic modeling of the electric field ahead of the tip of a lightning leader. Journal of Geophysical Research: Atmospheres, 122, 8120-8134. https://doi.org/10.1002/ 2016JD026206

Smith, D. M., Lopez, L. I., Lin, R. P., \& Barrington-Leigh, C. P. (2005). Terrestrial gamma-ray flashes observed up to 20 MeV. Science, 307 , 1085-1088.

Tavani, M., Marisaldi, M., Labanti, C., Fuschino, F., Argan, A., Trois, A., et al. (2011). Terrestrial gamma-ray flashes as powerful particle accelerators. Physical review letters, 106(1).

Tierney, D., Briggs, M., Fitzpatrick, G., Chaplin, V., Foley, S., McBreen, S., et al. (2013). Fluence distribution of terrestrial gamma ray flashes observed by the Fermi gamma-ray burst monitor. Journal of Geophysical Research: Space Physics, 118, 6644-6650. https://doi.org/10. 1002/jgra.50580

Xu, W., Celestin, S., \& Pasko, V. P. (2012). Source altitudes of terrestrial gamma-ray flashes produced by lightning leaders. Geophysical Research Letters, 39, L08801. https://doi.org/10.1029/2012GL051351

Xu, W., Celestin, S., \& Pasko, V. P. (2015). Optical emissions associated with terrestrial gamma ray flashes. Journal of Geophysical Research: Space Physics, 120, 1355-1370. https://doi.org/10.1002/2014JA020425

Xu, W., Celestin, S., Pasko, V. P., \& Marshall, R. A. (2017). A novel type of transient luminous event produced by terrestrial gamma-ray flashes. Geophysical Research Letters, 44, 2571-2578. https://doi.org/10.1002/2016GL072400 